\title{
Covariant gradient continua: wave propagation within heterogeneous material
}

\author{
G. FuthazaR ${ }^{a}$, L. Le Marrec and L.R. Rakotomanana \\ Institut de Recherche Mathématiques de Rennes, Université de Rennes 1, Campus Beaulieu, 35042 Rennes, France
}

Received 17 June 2013, Accepted 22 April 2014

\begin{abstract}
Riemann-Cartan geometry is used to model continuum with defects. In order to illustrate the differences induced by two possible definitions for the strain (spatial or material) in this framework, propagation of 3D waves is studied for a simple example of infinite continuum with uniform and stationary defects density. Anisotropy and attenuation are caught by both models even if these effects are quite different. Furthermore the material strain induces chirality and uniform breathing mode.
\end{abstract}

Key words: Gradient continuum / strain / heterogeneous wave propagation / dislocation

\section{Introduction}

The role played by geometry in physics is commonly acknowledged. In 1909, Cosserat brothers introduce continuum model involving independent field of rotation in addition to the displacement. Inspired by this approach Cartan has developed continuum models based on Riemann-Cartan (RC) manifold, endowed with not only the metric (measuring the shape change) but also an affine connection $\nabla$ which replaces the classical gradient operator $\bar{\nabla}[1]$. Affine connection is enriched by torsion tensor and gives rise to curvature tensor. If one of these later tensors is non-zero, the geometrical equivalence between the Euclidian space and the continuum is lost. Indeed, Cartan circuit brings new perspectives for modeling continuous distribution of dislocations and disclinations in the continuum [2-4]. According to Noll's definition: a continuum is said to be homogeneous if there exists a state of deformation of material manifold in which the mass density is uniform [5]. Following this approach, one of the authors proposes a class of non-homogenity allowing discontinuities of scalar fields and vector fields described by torsion and curvature [6]. Application to wave propagation in non-homogeneous continuum is developed in [7] under the hypothesis of covariant spatial strain for continuum.

Here we give a quick overview of main results obtained by the authors in [8]. We first recall the geometric concepts needed to describe the continuum and the link with dislocations. The RC geometry is used to derive the mechanical conservation laws. However, a fundamental discussion raises for the choice of covariant derivative to define the strain: Could we neglect or not the defect's

\footnotetext{
${ }^{a}$ Corresponding author: gregory.futhazar@gmail.com
}

influence on the deformation? That is to say should we use the symmetric connection $\bar{\nabla}$ or the full connection $\nabla$ to define the strain? In order to address this question both models are developed and compared in the framework of wave propagation. Details and discussion, can be found in $[8]$.

\section{Some differential geometry}

This section is a brief recall of definitions and results in differential geometry [9]. Greek indexes are related to coordinates in a material manifold $\mathbb{M}$ modeling the actual continuum whereas the latin ones refer to Euclidian space (with metric $g_{i j}$ ). $\mathbf{e}_{\mathbf{i}}$ (resp. $\mathbf{e}_{\mu}$ ) are the basis vectors of the tangent space of $\mathbb{E}$ (resp. $\mathbb{M})$. The transition between the two states is performed by the triads $e_{i}^{\mu}$ which relate for the local transformation of the $\mathbb{E}$ state into the $\mathbb{M}$-state: $\mathrm{d} x^{\mu}=e_{i}^{\mu} \mathrm{d} X^{i}$ (in classical elasticity $\left.e_{i}^{\mu}=\partial x^{\mu} / \partial X^{i}=\partial_{i} x^{\mu}\right)$. The material metric $g_{\mu \nu}$ and the reciprocal triad $e_{\mu}^{i}$ are given by: $g_{\mu \nu}=e_{\mu}^{i} e_{\nu}^{j} g_{i j}$, with $e_{i}^{\mu} e_{\mu}^{j}=\delta_{i}^{j}$, and where $\delta_{i}^{j}$ is the Kronecker symbol. Manifolds $\mathbb{E}$ and $\mathbb{M}$ are interpreted as two different states of the same body. $\mathbb{E}$-state is considered as a homogeneous continuum whereas the $\mathbb{M}$-state includes defects engendered by non-holonomic deformation [5]. Indeed, the differential structure of $\mathbb{M}$ is not necessarily Euclidian (Fig. 1). The covariant derivative of a basis vector $\mathbf{e}_{\beta}$ along the direction $\mathbf{e}_{\alpha}$ in the material coordinates is obtained by the affine connection $\nabla$ defined as $\nabla_{\alpha} \mathbf{e}_{\beta}=\Gamma_{\alpha \beta}^{\gamma} \mathbf{e}_{\gamma}$, with its connection coefficients in terms of triads: $\Gamma_{\alpha \beta}^{\gamma}=e_{i}^{\gamma} \partial_{\alpha} e_{\beta}^{i}=-e_{\beta}^{i} \partial_{\alpha} e_{i}^{\gamma}$. The connection is metric compatible $\nabla g=0$. The $\mathbb{M}$-state generalizes 

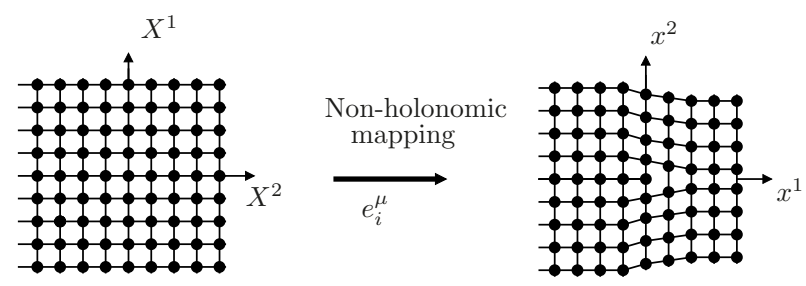

Fig. 1. Triads define new metric and covariant derivative on the material manifold.

the definition of continuum as it includes defects induced by non-holonomic transformations [5] so that its structure is not necessarily Euclidian (Fig. 1). Precisely, if triads do not verify the Schwartz integrability condition $\partial_{\alpha} e_{\beta}^{i}-\partial_{\beta} e_{\alpha}^{i} \neq 0$, the connection is not symmetric and gives rise to the torsion tensor:

$$
S_{\alpha \beta}^{\gamma}=\frac{1}{2}\left(\Gamma_{\alpha \beta}^{\gamma}-\Gamma_{\beta \alpha}^{\gamma}\right)=\frac{1}{2} e_{i}^{\gamma}\left(\partial_{\alpha} e_{\beta}^{i}-\partial_{\beta} e_{\alpha}^{i}\right)
$$

By theorem, a given metric uniquely defines symmetric connection under the $\nabla g=0$ assumption: the Levi-Civita connection $\bar{\nabla}$ with associated Christoffel coefficients $\bar{\Gamma}_{\alpha \beta}^{\gamma}$.

Manifold endowed with both curvature [9] and torsion is a Riemann-Cartan manifold. We can decompose the connection coefficient into two parts:

$$
\begin{aligned}
\Gamma_{\alpha \beta}^{\gamma} & =\bar{\Gamma}_{\alpha \beta}^{\gamma}+K_{\alpha \beta}^{\gamma}, \\
\text { where } \quad K_{\alpha \beta}^{\gamma} & =\frac{1}{2}\left(S_{\alpha \beta}^{\gamma}+g^{\gamma \lambda} S_{\lambda \beta}^{\kappa} g_{\kappa \alpha}+g^{\gamma \lambda} S_{\lambda \alpha}^{\kappa} g_{\kappa \beta}\right)(1)
\end{aligned}
$$

\section{Relation with dislocations}

In continuum mechanics theory, the chosen scale suggests us to deal with density of dislocations rather than dislocation lines. The fail of closure of displacement vector along a closed curve $C$ can be expressed in term of Euclidian components:

$$
\begin{aligned}
b^{i} & =\oint_{C} d x^{\alpha} \partial_{\alpha} X^{i}(x)=\oint_{C} d x^{\alpha} e_{\alpha}^{i} \\
& =\int_{\Sigma} d x^{\alpha} \wedge d x^{\beta} e_{\gamma}^{i} S_{\alpha \beta}^{\gamma}=\int_{\Sigma} d x^{\alpha} \wedge d x^{\beta} S_{\alpha \beta}^{i}
\end{aligned}
$$

Hence, the torsion tensor is interpreted as the surface density of Burgers vector. $\mathbf{b}$ is thought as sum of all Burgers vectors from dislocation lines going through the surface $\Sigma$. To conclude, a defective continuum should not be endowed with the differential structure of the Euclidian subspace it occupies.

\section{Navier equation in a Riemann-Cartan manifold}

We focus on a superimposed evolution of a continuum with defects (RC manifold) assuming small elastic perturbation which does not create new defects. We consider the same conservation equations as in classical elasticity but we need to define the differential operators by means of material connection attached to the (defective) reference state to capture the influence of defects during the superimposed motion. Let $\mathbf{u}, \varepsilon$ and $\sigma$ be respectively the superimposed displacement field, the small-strain tensor and the (symmetric) stress tensor. Then the conservation laws are e.g. [6] : (i) mass conservation $\rho=\rho_{0} \operatorname{det}\left(e_{i}^{\alpha}\right)$; and (ii) momentum conservation: $\rho_{0} \partial_{t}^{2} \mathbf{u}=\nabla \sigma$ (no external force). We assume a linear Hooke's law for an homogeneous isotropic elastic medium with constant Lamé coefficients $\lambda$ and $\mu: \sigma=\lambda \operatorname{Tr}(\varepsilon) \mathbf{I}+2 \mu \varepsilon$ where $\mathbf{I}$ is the identity tensor. We obtain the equation of propagation for the displacement:

$$
\rho_{0} \partial_{t}^{2} \mathbf{u}=\nabla[\lambda \operatorname{Tr}(\varepsilon) \mathbf{I}+2 \mu \varepsilon]
$$

At this step the definition of the strain tensor is questionable because two points of view can be adopted. According to the RC geometry of the reference state, as a first point of view, it seems more rigorous to use the covariant derivative $\nabla$ for calculating the gradient of the displacement. Indeed the strain tensor describes how the continuum is locally modified (i.e. the matter motion with respect to its neighbor). In this case, the gradient should use the material connection which, in addition to the metric, characterizes the geometrical structure of the material manifold e.g. [5]. From another slightly different but second point of view, we could suppose that the superimposed deformation is only sensible to the metric, hence the influence of defects on the strain definition would be neglected: each infinitesimal volume is deformed without further restriction due to the defective arrangement within matter. Adopting this second hypothesis, we can calculate the strain (as the symmetric part of the displacement gradient) with the help of the Levi-Civita connection $\bar{\nabla}$. Hereafter we derive equation (3) for each hypothesis.

\section{Spatial strain}

The spatial strain is defined with Levi-Civita connection $\varepsilon \equiv \frac{1}{2}\left[\bar{\nabla} \mathbf{u}+(\bar{\nabla} \mathbf{u})^{t}\right]$ and using the decomposition equation (1) with the notation $\nabla \sigma=\bar{\nabla} \sigma+K \sigma$, equation (3) becomes:

$$
\rho_{0} \partial_{t}^{2} \mathbf{u}=(\lambda+\mu) \bar{\nabla}(\bar{\nabla} \mathbf{u})+\mu \bar{\Delta} \mathbf{u}+\mathbf{f}^{\mathrm{sp}}
$$

where $\mathbf{f}^{\mathrm{sp}}$, depending on the torsion tensor $\mathbf{S}$, is an additional force due to defects:

$$
\mathbf{f}^{\mathrm{sp}}(\bar{\nabla} \mathbf{u}, \mathbf{S})=\lambda(\bar{\nabla} \mathbf{u})(K \mathbf{I})+\mu K\left(\bar{\nabla} \mathbf{u}+(\bar{\nabla} \mathbf{u})^{t}\right)
$$

\section{Material strain}

Here small-strain tensor is defined by means of material connection $\varepsilon:=\frac{1}{2}\left[\nabla \mathbf{u}+(\nabla \mathbf{u})^{t}\right]$. It is now designated as material strain. Then defining the convention $\nabla \mathbf{u}=\bar{\nabla} \mathbf{u}+K \mathbf{u}$, the wave equation is:

$$
\rho_{0} \partial_{t}^{2} \mathbf{u}=(\lambda+\mu) \bar{\nabla}(\bar{\nabla} \mathbf{u})+\mu \bar{\Delta} \mathbf{u}+\mathbf{f}^{\text {mat }}
$$



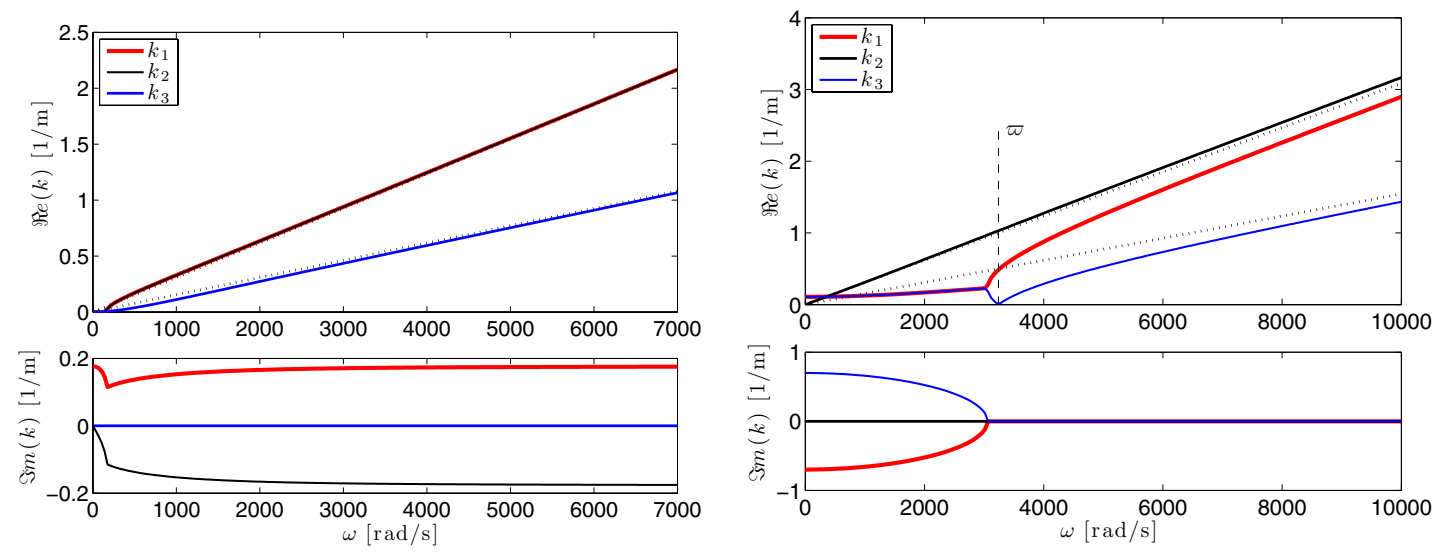

Fig. 2. Dispersion curves $\Re e(k(\omega))$ (bottom) and $\Im m(k(\omega))$ (top) for a propagation along $\phi=\pi / 3$. Left: spatial strain, right: material strain. Roots are labeled arbitrarily $k_{i=1,2,3}$. Doted lines: classical dispersion curves $k_{l}=\omega / c_{l}$ and $k_{t}=\omega / c_{t}$. Numerical values: $S=1 \mathrm{~m}^{-1}, \rho_{0}=7500 \mathrm{~kg} . \mathrm{m}^{-3}$, Young modulus $E=210 \mathrm{GPa}$, Poisson ratio $\nu=1 / 3$.

with a force $\mathbf{f}^{\text {mat }}$ added to the classical Navier equation:

$$
\begin{aligned}
\mathbf{f}^{\mathrm{mat}}= & \mathbf{f}^{\mathrm{sp}}+\lambda(\bar{\nabla}(K \mathbf{u})+(K \mathbf{u})(K \mathbf{I})) \\
& +\mu\left(\bar{\nabla}\left(K \mathbf{u}+(K \mathbf{u})^{t}\right)+K\left(K \mathbf{u}+(K \mathbf{u})^{t}\right)\right)
\end{aligned}
$$

The forces $\mathbf{f}^{\mathrm{sp}}$ and $\mathbf{f}^{\text {mat }}$ are configurational forces like Peach-Koehker forces [10]. Other formulation of equation (5) highlights the influence of Ricci curvature [8].

\section{Illustration and discussion for a simple torsion density}

We focus on a simple example. A uniform distribution of screw dislocation within an infinite continuum is modeled by a constant torsion density, so that $S_{j k}^{i}=0$ except $S_{23}^{1}=-S_{23}^{1}=S$. Furthermore for sake of simplicity, we work with Cartesian coordinates which implies $g_{i j}=\delta_{i j}$. Under these hypotheses we can show that:

$$
\begin{gathered}
\mathbf{f}^{\mathrm{sp}}=\mu S\left(\begin{array}{c}
0 \\
\partial_{1} u_{3}+\partial_{3} u_{1} \\
-\left(\partial_{1} u_{2}+\partial_{2} u_{1}\right)
\end{array}\right) \\
\mathbf{f}^{\mathrm{mat}}=\mu S\left(\begin{array}{c}
\partial_{2} u_{3}-\partial_{3} u_{2} \\
\partial_{3} u_{1}+2 \partial_{1} u_{3}-S u_{2} \\
-\left(2 \partial_{1} u_{2}+\partial_{2} u_{1}+S u_{3}\right)
\end{array}\right)
\end{gathered}
$$

At this level we note that the spatial model is not an approximation of the material ones where $\mathcal{O}\left(S^{2}\right)$-contribution would be neglected for small density of defects.

\section{Particular solution: breathing modes}

Injecting an uniform $\mathbf{u}$ in equation (5) implies $u_{1}=$ cste and $\left(\partial_{t}^{2}+\left(c_{t} S\right)^{2}\right) u_{i}=0$ for $i=2,3$ where $c_{t}=$ $\sqrt{\mu / \rho_{0}}$. Time-harmonic solutions are:

$$
u_{2}=U_{2} e^{i \varpi t}, \quad u_{3}=U_{3} e^{i \varpi t}, \quad \text { with } \quad \varpi=c_{t} S .
$$

This vibration with infinite wavelength is sometimes called breathing. In other words, spatial dependence is governed by macroscopic geometry (and boundary condition) whereas frequency is induced by the microstructure. In this typical example, the eigen-frequency is independent of the size of the specimen and all the domain oscillates in phase (but some boundary conditions can annealed this phenomena). This breathing is not observed if the spatial strain is used.

\section{Plane-waves eigenfunctions}

We are interested in plane-wave solutions $\mathbf{u}(\mathbf{x}, t)=$ $\mathbf{U} e^{i(k \mathbf{n} \mathbf{x}-\omega t)}$ for which we developped dispersion relation between the wavenumber and the frequency $\omega$. This relation and the polarization $\mathbf{U}$ depend on the direction of propagation $\mathbf{n}$. By invariance of the problem by rotation around $\mathbf{e}_{\mathbf{1}}$-axis and symmetry to the $\left(\mathbf{e}_{\mathbf{2}}, \mathbf{e}_{\mathbf{3}}\right)$-plane, we restrict our resolution to $\mathbf{n x}=\cos \phi x_{1}+\sin \phi x_{2}$ with $0 \leq \phi \leq \frac{\pi}{2}$.

Figure 2 shows the real part and the attenuation for both models and propagation along $\phi=\pi / 3$. We see two transversal and one longitudinal waves. Models show similar behavior for high frequency and the main difference is for the attenuation. Spatial model shows uniform attenuation for sufficiently high frequency. The material case shows decreasing attenuation with frequency which is qualitatively obtained by other models with uniform distribution of discrete dislocations [11,12]. Experimental results focusing on waves in continuum with dislocations would be helpful here.

\section{High frequency regime}

In the high frequency regime, a first-order Taylor expansion is used around $\varpi / \omega=0$ to give asymptotic form 


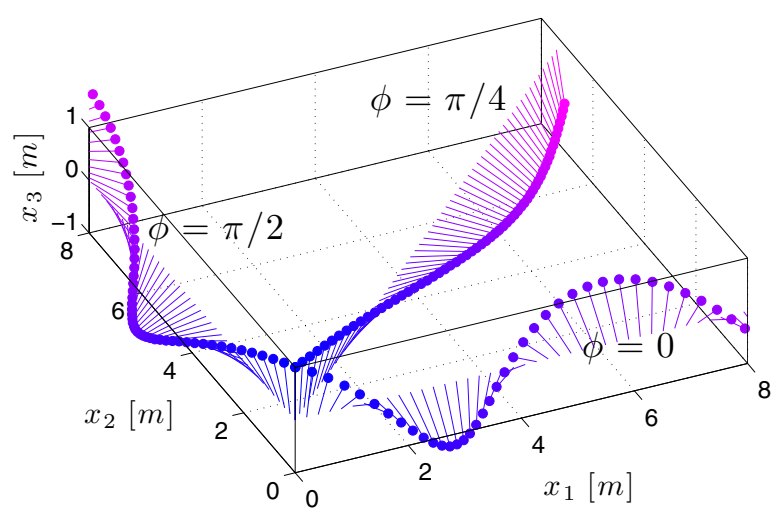

Fig. 3. Polarization $\mathbf{V}_{\mathbf{1}}$ during propagation along three directions (material case). The polarization is adjusted to be $\mathbf{e}_{\mathbf{3}}$ at the origin. Numerical values of Figure 2 and $\omega=10^{5} \mathrm{rad}_{\mathrm{s}}{ }^{-1}$.

of $k_{i}$. With $k_{t}$ the wavenumber of transversal waves in homogeneous continuum, eigen-solution of equation (5) can be writen as:

$$
\begin{aligned}
\mathbf{v}^{\mathbf{1}} & =\left(\cos (\boldsymbol{\kappa} \mathbf{x}) \mathbf{e}_{\theta}-\sin (\boldsymbol{\kappa} \mathbf{x}) \mathbf{e}_{\mathbf{z}}\right) e^{i\left(\mathbf{k}_{\mathbf{t}} \mathbf{x}-\omega t\right)} \\
& :=\mathbf{V}_{\mathbf{1}}(\mathbf{x}) e^{i\left(\mathbf{k}_{\mathbf{t}} \mathbf{x}-\omega t\right)} \\
\mathbf{v}^{\mathbf{2}} & =\left(\sin (\boldsymbol{\kappa} \mathbf{x}) \mathbf{e}_{\theta}+\cos (\boldsymbol{\kappa} \mathbf{x}) \mathbf{e}_{\mathbf{z}}\right) e^{i\left(\mathbf{k}_{\mathrm{t}} \mathbf{x}-\omega t\right)} \\
& :=\mathbf{V}_{\mathbf{2}}(\mathbf{x}) e^{i\left(\mathbf{k}_{\mathbf{t}} \mathbf{x}-\omega t\right)}
\end{aligned}
$$

For material strain $\kappa=\frac{S}{4}(1+3 \cos 2 \phi)$. The shear wave has a chiral structure: as the wave propagates, its polarization $\left(\mathbf{V}_{\mathbf{1}}\right.$ or $\left.\mathbf{V}_{\mathbf{2}}\right)$ regularly turns about the propagation's axis. Note that $\kappa$, and then the speed of rotation, is a function of the direction of propagation $\phi$ : a clockwise rotation governs the polarization for $0 \leq \phi \leq \phi_{c}$ whereas it is counterclockwise for $\phi_{c} \leq \phi \leq \pi / 2$, with $\phi_{c} \sim 54.7^{\circ}$ (Fig. 3).

For spatial strain $\kappa=\frac{S}{2} \cos \phi \sqrt{\cos 2 \phi}$ is complex inducing spatial attenuation which would avoid the chiral structure.

\section{Conclusions}

In the $\mathrm{RC}$ framework, defects are modeled with intrinsic differential operators taking into account torsion and metric. In this context we discussed the definition of strain tensor. A priori, the material connection is more rigorous because it takes into account the geometric structure of the material manifold (defective reference state) on the strain. For both definitions we developped generalized Navier equations (4) and (5). In each case, we exhibit configurational forces $\mathbf{f}^{\text {sp }}$ and $\mathbf{f}^{\text {mat }}$. The spatial one is not an approximation of the material one where $\mathcal{O}\left(S^{2}\right)$ would be neglected. Therefore the effects caught in the material are not due to large density of defects which is not an argument to neglect their influence on the strain. To illustrate the difference between both models, we consider infinite continuum with uniform density of screw dislocations. In the material case, the $\mathcal{O}\left(S^{2}\right)$-terms induce a stiffness operator which may imply breathing modes for the continuum. Furthermore both definitions imply anisotropy for the wave propagation however the dispersion and the attenuation are qualitatively different. Material strain model shows no attenuation for high frequency which has been observed with discrete model of dislocation. The material case shows chirality effect which could be observed along a large distance and be used to measure the density of defects in a continuum. This phenomenon is spatially attenuated for the spatial strain. Discussion and comparison with other models could be enriched by considering a full perturbation of the connection: strain and defect density.

\section{References}

[1] E. Cartan, On Manifolds with an Affine Connection and the Theory of General Relativity, Bibliopolis, Napoli, 1986 (English translation of the French original by A. Magnon and A. Ashtekar).

[2] B.A. Bilby, R. Bullough, E. Smith, Continuous distributions of dislocations: a new application of the method of non-Riemannian geometry, Proc. R. Soc. Lond. A 231 (1955) 263-273

[3] K. Kondo, Non-Riemannian geometry of imperfect crystals from a macroscopic viewpoint, in Memoirs of the unifying study of basic problems in engineering and physical science by means of geometry, Gakujutsu Benken Fukyu-Kai, Tokyo, 1955, Vol. 1

[4] E. Kröner, Continuum theory of defects, in "Physics of defects" (Les Houches, Session 35), edited by R. Balian et al., North-Holland, Amsterdam, 1980, pp. 215-315

[5] W. Noll, Materially uniform simple bodies with inhomogeneities, Arch. Rat. Mech. Anal. 27 (1967) 1-32

[6] L.R. Rakotomanana, Contribution à la modélisation géométrique et thermodynamique d'une classe de milieux faiblement continus, Arch. Rat. Mech. Anal. 141 (1997) 199-236

[7] N.A. Tamarasselvame, M. Buisson, L.R. Rakotomanana, Wave propagation within some non-homogeneous continua, C. R. Mécanique 339 (2011) 779-788

[8] G. Futhazar, L. Le Marrec, L. R. Rakotomanana, Covariant gradient continua applied to wave propagation within defected material, Archive of Applied Mech. 84 (2014) 1339-1356

[9] M. Nakahara, Geometry, Topology and Physics, Graduate Student Series Physics, IOP Publishing, 1996, pp. 263-326

[10] G.A. Maugin, Configurational forces: thermomechanics, physics, mathematics, and numerics, Taylor \& Francis, 2010

[11] A. Maurel, J-F. Mercier, F. Lund, Scattering of an elastic wave by a single dislocation, J. Acoust. Soc. Am. 115 (2004) $2773-2780$

[12] A. Maurel, J-F. Mercier, F. Lund, Elastic wave propagation through a random array of dislocations, Phys. Rev. B 70 (2004) 024303 